\title{
A Distributed Secondary Control Strategy for Power Sharing and Voltage Regulation by Multiple Converters in DC Micro-grids
}

\author{
Shahid Ullah ${ }^{\mathrm{a}}$ \\ Department of Electrical \& Electronics \\ Faculty of Engineering \\ aUniversiti Malaysia Sarawak \\ Kota Samarahan, Sarawak, Malaysia
}

\author{
Ahmed M. A. Haidar ${ }^{a b}$ \\ aUniversiti Malaysia Sarawak \\ Kota Samarahan, Sarawak, Malaysia \\ bUniversity of Southern Queensland \\ Toowoomba, Queensland, Australia
}

\author{
Kashem M. Muttaqic ${ }^{\mathrm{c}}$, Tony Ahfock ${ }^{\mathrm{b}}$ \\ School of Electrical, Computer, and \\ Telecommunications Engineering \\ 'University of Wollongong \\ Wollongong, NSW, Australia
}

\begin{abstract}
Recent research and advancement in DC systems reveal that DC distribution may be an efficient, reliable and economical solution to avoid the inherent issues associated with AC power systems such as frequency control, harmonics and synchronization. However, some key operational issues in DC micro-grids are to ensure a stable output voltage and proper load sharing among the sources. Improper current sharing and voltage variations in the conventional droop control causes circulating currents due to the presence of droop and line resistance between converters. This paper presents a distributed secondary control strategy to smooth the output voltage and maintain power sharing based on average current/voltage control and circulating current minimization. Further, an additional current feedback loop is introduced to modify the micro-grid reference voltage during overload conditions for minimizing the line voltage drop and power losses. The conventional communication links are used for exchanging information to the relevant controllers simultaneously to implement the proposed distributed control. The performance of the proposed approach has been verified by simulating two parallel Buck converters supplying a common load. The effect of load switching on voltage regulation and power sharing has also been analyzed.
\end{abstract}

Keywords- DC micro-grid, DC-DC converters, multi-tap reference voltage, power sharing.

\section{INTRODUCTION}

The advancement in power electronics made the power conversion, control and level transformation more efficient and encouraged researchers to reconsider DC micro-grids and DC distribution systems for efficient integration of renewable energy sources (RES). More than $10 \%$ of energy saving can be obtained by using the DC distribution when compared to $\mathrm{AC}$ distribution where inverters are used. DC micro-grids can better utilize RES with natively DC output, energy storage systems (ESS) and the change in loads. This will eliminate the major challenges in $\mathrm{AC}$ micro-grids such as synchronization, frequency control, reactive power regulation, and power quality [1]. Furthermore, DC systems reduce the weight of distribution network up to 10 tons/MW because of the higher power carrying capacity of DC cables compared to $\mathrm{AC}$ cables and lesser losses in power converters compared to AC transformers. Such characteristics make DC systems more suitable for applications such as electric aircraft and electric vehicle [2]. Recently, low-voltage DC microgrids have been deployed in telecommunication, data centers, and traction systems. However, with the increasing deployment and the emerging challenges of DC micro-grid control, efficient and robust control techniques must be

This work has been supported by a grant (F02/FRGS/1497/2016) from the Ministry of Higher Education (Malaysia). developed for DC systems to compete with the established AC systems [1], [3].

To date, various control approaches were reported in the literature. Generally, a three-level hierarchical control strategy (primary, secondary, and tertiary control) has been developed for standardized operation and better functionality of micro-grids. At the primary level of this hierarchical control, power sharing and rapid voltage stabilization are ensured. For this purpose, typically droop control is adopted. Secondary control compensates the steady state voltage deviations imposed by primary control. Power management and optimization in DC micro-grids are achieved at the tertiary level of hierarchical control. The main control objective in DC micro-grids is the simultaneous output voltage stability and proper load power sharing among the sources, irrespective of the load or source power variations. Output voltage fluctuations of power converters and mismatch between line resistances of different sources can degrade the power sharing and voltage stability in DC microgrids. The traditional droop control provides better current sharing in DC micro-grids; however, droop control imposes a voltage drop due to the presence of droop resistances. The value of droop resistance is directly proportional to current sharing accuracy of droop control. Therefore, higher droop resistance will impose more voltage deviations in the output voltage. Moreover, high voltage deviations may take place due to the high load currents or mismatch between line resistances. To enhance the voltage stability of DC microgrid, such voltage deviations should be eliminated. Another strategy that combines the primary and secondary levels of hierarchical control relies on advanced decentralized modelbased control approaches [4]-[9].

Among the existing droop control strategies in DC microgrids, an adaptive droop control technique to overcome the limitations of traditional droop control was proposed in [10], which provides improved current sharing accuracy and output voltage regulation simultaneously. Authors in [11] proposed an observer-based control strategy for DC micro-grid that combines the droop control with current feed-forward control. This technique can effectively improve the DC voltage stability during load variations, change in cable impedances or alterations in droop control gains. A sliding mode controller was introduced by [12] to ensure accurate load sharing with improved voltage stability in all operating conditions of DC micro-grid. Authors in [13] presented a distributed control to improve the drawback of the conventional hierarchical control approach. Here, the droop control method was facilitated with an average current sharing control in a decentralized fashion by implementing 